\title{
The novel mTOR inhibitor Torin-2 induces autophagy and downregulates the expression of UHRF1 to suppress hepatocarcinoma cell growth
}

\author{
CONGREN WANG ${ }^{1 *}$, XUEJIN WANG $^{2 *}$, ZIJIAN SU $^{1}$, HONGJIANG FEI $^{1}$, XIAOYU LIU $^{1}$ and QUNXIONG PAN ${ }^{1}$ \\ ${ }^{1}$ Department of Surgical Oncology, The First Hospital of Quanzhou Affiliated to Fujian Medical University; \\ ${ }^{2}$ Department of Obstetrics and Gynecology, The Second Affiliated Hospital of \\ Fujian Medical University, Fujian, Quanzhou 362000, P.R. China
}

Received March 10, 2015; Accepted April 29, 2015

DOI: $10.3892 /$ or.2015.4146

\begin{abstract}
Mammalian target of rapamycin (mTOR) is frequently upregulated in hepatocellular carcinoma (HCC). Blockage of mTOR was found to induce marked reduction in HCC growth in preclinical models. In the present study, we tested a novel mTOR inhibitor, Torin-2, for its antitumor efficacy in HCC cell lines Hep G2, SNU-182 and Hep 3B2.1-7. The HCC cell lines were cultured in vitro. These cells were treated with Torin-2. Cell apoptosis was evaluated by Annexin V staining. Cell proliferation and cell cycle progression were determined by Ki67 staining and propidium iodide staining, respectively. mTOR signaling, autophagy induction and expression of ubiquitin-like containing PHD and RING finger domains 1 (UHRF1) were assessed by western blot analysis. The UHRF1 mRNA level was determined by real-time PCR. We found that Torin-2 effectively suppressed the growth and survival of HCC cell lines, demonstrated by reduced proliferation and a high rate of apoptosis. Further study elucidated that in addition to blocking mTOR complex 1 (mTORC1)-associated cell cycle progression and induction of autophagy, Torin-2 downregulated transcription of UHRF1, an essential regulator of DNA methylation that is highly expressed in HCC cell lines. Consistently, the level of DNA (cytosine-5)-methyltransferase 1 (DNMT1) was higher after treatment of the HCC cell lines with Torin-2. The downregulation of UHRF1 by Torin-1 was partially due to a decrease in the UHRF1 mRNA level. Torin-2 effectively inhibited HCC cell proliferation through induction of autophagy. Torin-2-induced downregulation of UHRF1 expression may also contribute to
\end{abstract}

Correspondence to: Dr Qunxiong Pan, Department of Surgical Oncology, The First Hospital of Quanzhou Affiliated to Fujian Medical University, 250 East Road, Fujian, Quanzhou 362000, P.R. China E-mail: qunxiongpan@126.com

*Contributed equally

Key words: mammalian target of rapamycin, hepatocellular carcinoma, Torin-2, autophagy, tumor growth its antitumor effect. Our research provides new clues regarding the antitumor effects of Torin-2 and sheds light on a novel therapeutic approach for HCC.

\section{Introduction}

Hepatocellular carcinoma (HCC) is the third leading cause of cancer-related mortality worldwide (1). Advances are being made in understanding the mechanisms underlying HCC, which in turn could lead to novel therapeutics. Signaling pathways have become a major source of targets for novel therapies for HCC. Multiple signaling modules are deregulated in HCC, including some related to growth factor signaling such as IGF, EGF, PDGF, FGF and HGF (2) and cell differentiation such as WNT, Hedgehog and Notch (3) and angiogenesis (VEGF) (4). Intracellular mediators such as RAS and Akt/mTOR may also play a role in HCC development and progression (5-7). Different molecular mechanisms have been shown to induce aberrant pathway activation. These include point mutations, chromosomal aberrations and epigenetically driven downregulation.

Among the above-mentioned signaling pathways, the $\mathrm{PI} 3 \mathrm{~K} / \mathrm{Akt} / \mathrm{mTOR}$ pathway has received much attention from researchers due to its critical role in tumor cell proliferation, differentiation, survival, motility and invasion (8). Growth factors and hormones activate a protein known as PI3K, which in turn activates Akt, which then activates the mammalian target of rapamycin (mTOR) by inactivating TSC2 to prevent inhibition of mTOR complex 1 (mTORC1). Active mTORC1 has a number of downstream biological effects including translation of mRNA via the phosphorylation of downstream targets (4E-BP1 and p70 S6 kinase), suppression of autophagy and activation of transcription leading to mitochondrial metabolism or adipogenesis. Evidence also indicates that the mTORC1 complex plays a role in HCC progression (9). Numerous studies have been performed to target mTOR signaling to impact tumor biology especially for proliferation and survival. Rapamycin (sirolimus) is an mTOR inhibitor that has been shown to have antitumor properties (10). Everolimus is another mTOR inhibitor that has been shown to have activity against $\mathrm{HCC}$ in xenografts $(11,12)$. 
Torin-2 is a novel, second-generation ATP-competitive inhibitor that is potent and selective for mTOR with a superior pharmacokinetic profile to previous inhibitors $(13,14)$. Torin-2 inhibits mTORC1-dependent T389 phosphorylation on S6K (RPS6KB1). Torin-2 also exhibits potent biochemical and cellular activity against PIKK family kinases including ATM, ATR and DNA-PK. Torin-2 also displayed marked antiproliferative activity across a panel of cancer cell lines $(14,15)$. Cancer cell treatment with Torin-2 for $24 \mathrm{~h}$ resulted in a prolonged blockage of negative feedback and consequent T308 phosphorylation of Akt. These effects were associated with strong growth inhibition in vitro. Studies of Torin-2 suggest that it has potent antitumor activity and potential efficacy in HCC treatment.

Epigenetic regulation has emerged as an important mechanism that regulates many chromatin template-based processes, including transcription, DNA replication and repair (16). An important component of epigenetic regulation is DNACpG methylation. DNACpG methylation has been shown to play an important role in tumorigenesis (17). Ubiquitin-like containing PHD and RING finger domains 1 (UHRF1) has been found to effectively regulate DNACpG methylation, heterochromatin function and gene expression $(18,19)$. Overexpression of UHRF1 has been suggested to contribute to tumorigenesis including HCC $(20,21)$. However, the relationship between mTORC1 signaling and UHRF1-regulated DNACpG methylation has not been elucidated.

In the present study, we tested the effect of Torin-2 on proliferation, cell cycle progression and apoptosis of different hepatocellular carcinoma cell lines, Hep G2, SNU-182 and Hep 3B2.1-7. We found higher mTORC1 activity in all three cell lines, and consequently Torin-2 blocked mTORC 1 in a dose-dependent manner. Torin-2 treatment profoundly inhibited HCC cell proliferation and survival through autophagy induction. Unexpectedly, we discovered that Torin-2 also downregulated expression of UHRF1. UHRF1 transcription was partially decreased by Torin-2. Collectively, our research provides new clues regarding the anti-HCC effect of Torin-2 and sheds light on a novel therapeutic approach for HCC.

\section{Materials and methods}

Cell culture and treatment. Hep G2 and Hep 3B2.1-7 cells were cultured in Eagle's minimum Essential medium (DMEM) supplemented with $10 \%$ fetal bovine serum (FBS), $100 \mu \mathrm{g} / \mathrm{ml}$ penicillin, $50 \mu \mathrm{g} / \mathrm{ml}$ streptomycin and $2 \mathrm{mM}$ L-glutamine. SNU-182 cells were cultured in RPMI-1640 medium supplemented with $10 \%$ FBS, $100 \mu \mathrm{g} / \mathrm{ml}$ penicillin, $50 \mu \mathrm{g} / \mathrm{ml}$ streptomycin and $2 \mathrm{mM}$ L-glutamine. Torin- 2 was purchased from Selleck Chemicals (Houston, TX, USA) and was prepared following the manufacturer's instructions. Cells were treated with Torin-2 at indicated concentrations for indicated time-periods before they were subject to subsequent analysis. For growth curve assay, 20,000-30,000 cells were seeded into each well of 6-well plates. At the indicated time-points, the cells were trypsinized with $0.25 \%$ trypsin-EDTA, and the cell number was counted on a hemacytometer.

Apoptosis assay. Floating cells were collected after treatment. Adherent cells were incubated in $2 \mathrm{mM}$ ethylene diamine tetraacetic acid (EDTA)-PBS. Cells were gently flushed with a pipette to dissociate cells from the bottom of the wells. Flushed cells were pooled with floating cells. Cells were then centrifuged and stained with PI and Annexin V (both from BD Biosciences, Franklin Lakes, NJ, USA). Briefly, the cells were washed with PBS twice and then incubated in $100 \mu \mathrm{l}$ of binding buffer containing Annexin V-FITC (1:20) and $5 \mu \mathrm{g} / \mathrm{ml}$ PI. Four hundred microliters of binding buffer was added to each sample. Apoptosis was analyzed by flow cytometry within $1 \mathrm{~h}$.

Cell proliferation and cell cycle analysis. For cell proliferation analysis, the cells were stained with $5 \mu \mathrm{g} / \mathrm{ml}$ FITC-conjugated antibody against Ki67 (Abcam, Cambridge, MA, USA). For cell cycle analysis, the cells were fixed with $70 \%$ ethanol and stained with PI (40 $\mu \mathrm{g} / \mathrm{ml}$; BD Biosciences), according to the manufacturer's instructions. DNA content was analyzed on a BD LSRII flow cytometer.

Western blot analysis. The cell lysate was prepared by homogenization in RIPA buffer [20 mM Tris- $\mathrm{HCl}$, pH 7.5, $150 \mathrm{mM}$ $\mathrm{NaCl}, 1 \mathrm{mM} \mathrm{Na}{ }_{2}$ EDTA, $1 \mathrm{mM}$ ethylene glycol tetraacetic acid (EGTA), 1\% NP-40, 1\% sodium deoxycholate] and lysed on ice for $30 \mathrm{~min}$. The protein amount was quantified using Pierce $660 \mathrm{~nm}$ Protein Assay (Thermo Scientific, Waltham, MA, USA) following the manufacturer's instructions. Total protein from each sample was loaded onto $10 \%$ SDS-PAGE gel and electrophoresis was performed. For LC3B, proteins were loaded onto $4-20 \%$ Mini-Protean ${ }^{\circledR}$ TGX $^{\text {TM }}$ precast gels (Bio-Rad, Hercules, CA, USA). Proteins were then transferred onto nitrocellulose membranes and blocked with $5 \%$ non-fat dry milk and PBS for $1 \mathrm{~h}$ at room temperature (RT). After 3 washes with $0.05 \%$ Tween-20 PBS (PBST), the membranes were incubated with primary antibodies overnight at $4^{\circ} \mathrm{C}$. Antibodies against LC3B (D11), Beclin-1 (D40C5) and p62 (D5E2) were purchased from Cell Signaling Technology (Danvers, MA, USA). Membranes were washed 3 times with PBST and then incubated with horseradish peroxidase-conjugated secondary antibodies for $1 \mathrm{~h}$ at RT. Membranes were developed with SuperSignal West Pico Chemiluminescent Substrate (Thermo Scientific) and were visualized and the optical density of the identified protein bands on membranes was analyzed using a Biospectrum 500 Imaging System (UVP LLC, Upland, CA, USA).

Real-time PCR. Total RNAs were extracted using TRIzol and were reversely transcribed to cDNAs using SuperScript ${ }^{\circledR}$ III First-Strand Synthesis system according to the manufacturer's instructions. Real-time PCR was performed using Fast SYBR $^{\circledR}$-Green Master Mix on a 7300 Real-Time PCR system. Reagents and instruments were purchased from Invitrogen Life Technologies (Carlsbad, CA, USA). Data were analyzed with 7300 System software. GAPDH was used as an internal control for mRNA. Relative expression was calculated with respect to the control. The results are expressed as $2^{-\Delta \Delta \mathrm{Ct}}$. Primer sequences for each gene were: UHRF1 forward, 5'-CCAGCAGAGCAGCCTCATC-3' and reverse, 5'-TCC TTGAGTGACGCCAGGA-3'; GAPDH forward, 5'-TGATGA CATCAAGAAGGTGGTGAAG-3' and reverse, 5'-TCCTTG GAGGCCATGTGGGCCAT-3'. 

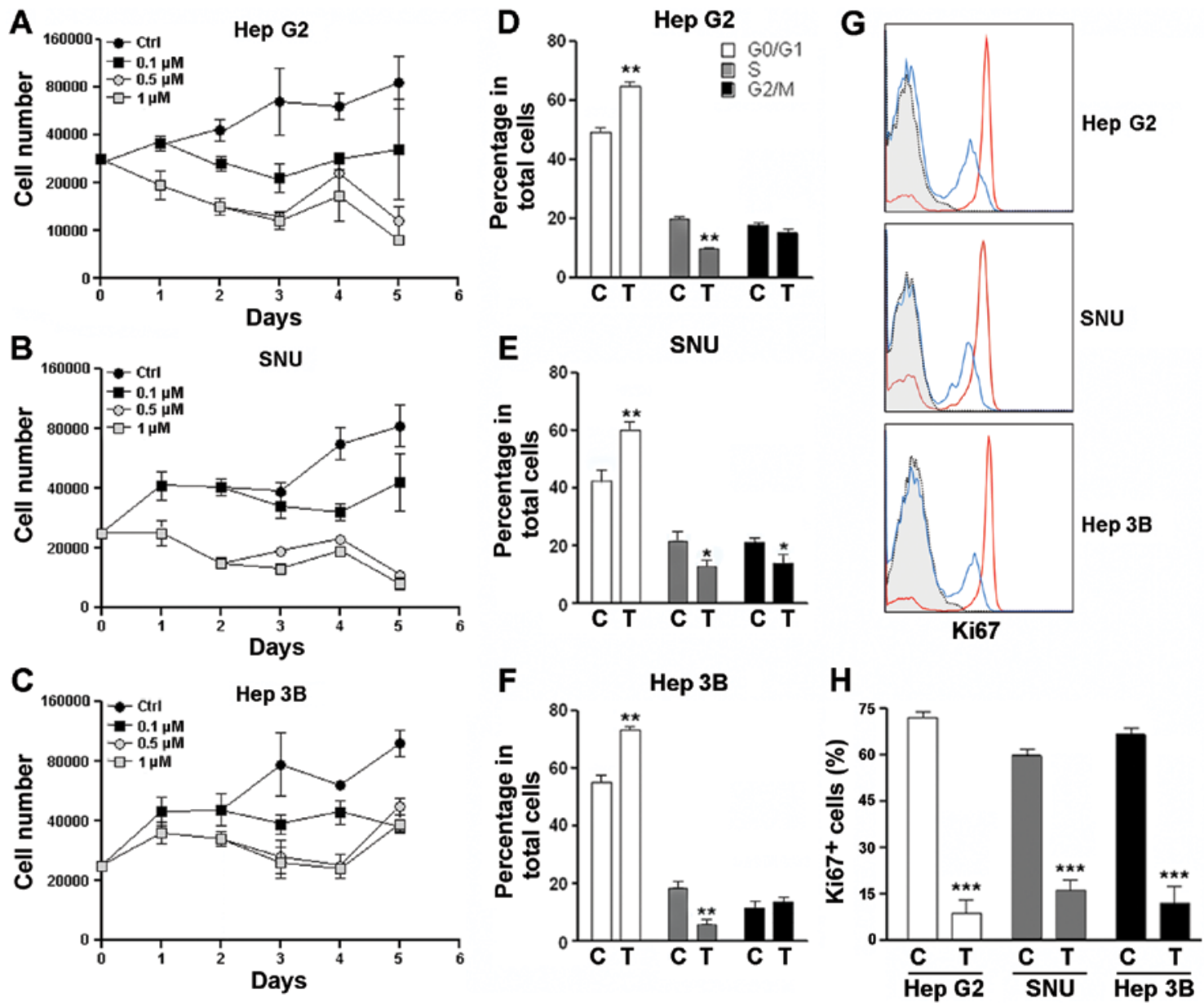

Figure 1. Torin-2 inhibits the proliferation of HCC cell lines. (A-C) Growth curves for the HCC cell lines after Torin-2 treatment. (D-F) Cell cycle analysis with PI staining for HCC cell lines on day 3 after Torin-2 treatment. (G) Representative histograms of Ki67 staining for HCC cell lines. Dotted line, isotype control. Red line, vehicle-treated cells. Blue line, $1 \mu \mathrm{M}$ Torin-2-treated cells. (H) Quantification of Ki67 staining. All experiments were repeated 4 times independently. $\mathrm{n}=8$ /group. SNU, SNU-182; Hep 3B, Hep 3B2.1-7; C, vehicle control; T, $1 \mu \mathrm{M}$ Torin-2-treated cells. ${ }^{*} \mathrm{P}<0.05,{ }^{* * *} \mathrm{P}<0.01$ and ${ }^{* * * *} \mathrm{P}<0.001$, in comparison with the vehicle control; HCC, hepatocellular carcinoma; PI, propidium iodide.

Statistical analysis. Data were analyzed and the results are presented as the mean \pm standard deviation. Student's t-test or one-way ANOVA was used for comparison of the mean between the groups and $\mathrm{P}<0.05$ was considered to indicate a statistically significant result.

\section{Results}

Torin-2 inhibits the proliferation of HCC cell lines. To determine whether Torin-2 impacts cancer cell biology, we firstly measured the growth curves of three HCC cell lines Hep G2, SNU-182 and Hep 3B2.1-7. As shown in Fig. 1A-C, Torin-2 reduced HCC cell growth in a dose-dependent manner and $1 \mu \mathrm{M}$ Torin-2 significantly inhibited the cell growth of all three cell lines tested. Torin-2 $(0.5 \mu \mathrm{M})$ exhibited similar but relatively weaker inhibitory effects. The inhibitory effect lasted for 4 to 5 days. Cell cycle analysis with PI staining indicated that on day 3 post-treatment, $1 \mu \mathrm{M}$ Torin- 2 induced significant $\mathrm{G}_{0} / \mathrm{G}_{1}$ phase arrest of the three cell lines, while the percentage of cells in the $\mathrm{S}$ phase were markedly reduced, suggesting that cancer cell proliferation was decreased by Torin-2 (Fig. 1D-F). Ki67 staining, which is a marker of cell cycle progression, also demonstrated that a lower percentage of cancer cells entered the cell cycle on day 3 after Torin-2 treatment (Fig. 1G and H). Collectively, our data indicated that Torin-2 effectively decreased cancer cell growth in our experimental settings.

Torin-2 blocks mTORC1 signaling to inhibit cancer cell proliferation. It has been reported that Torin-2 is an mTORC1 signaling inhibitor (14) and mTORC1 signaling has been shown to be important for tumor cell growth and metabolism (8). To ascertain whether the above anti-proliferative effect of Torin-2 is related to the blockage of mTORC1 signal transduction, we assessed activated phosphorylation of mTORC1 components using western blot analysis. Firstly, we confirmed that mTORC1 signaling was significantly activated in all three cancer cell lines in comparison with normal liver tissues, demonstrated by a higher phosphorylation level of mTOR and mTORC1 target proteins 4EBP1 and p70S6K (Fig. 2A). Torin-2 decreased activating phosphorylation of mTOR dose-dependently, suggesting that, similar to leukemic cells, Torin-2 effectively blocks mTORC1 signaling (Fig. 2B). In association with less mTORC1 activation, cyclin D1 expression was profoundly reduced in the three cell lines by Torin-2 dose-dependently (Fig. 2C-F). Cyclin D is critical for $\mathrm{G}_{1} / \mathrm{S}$ tran- 
A
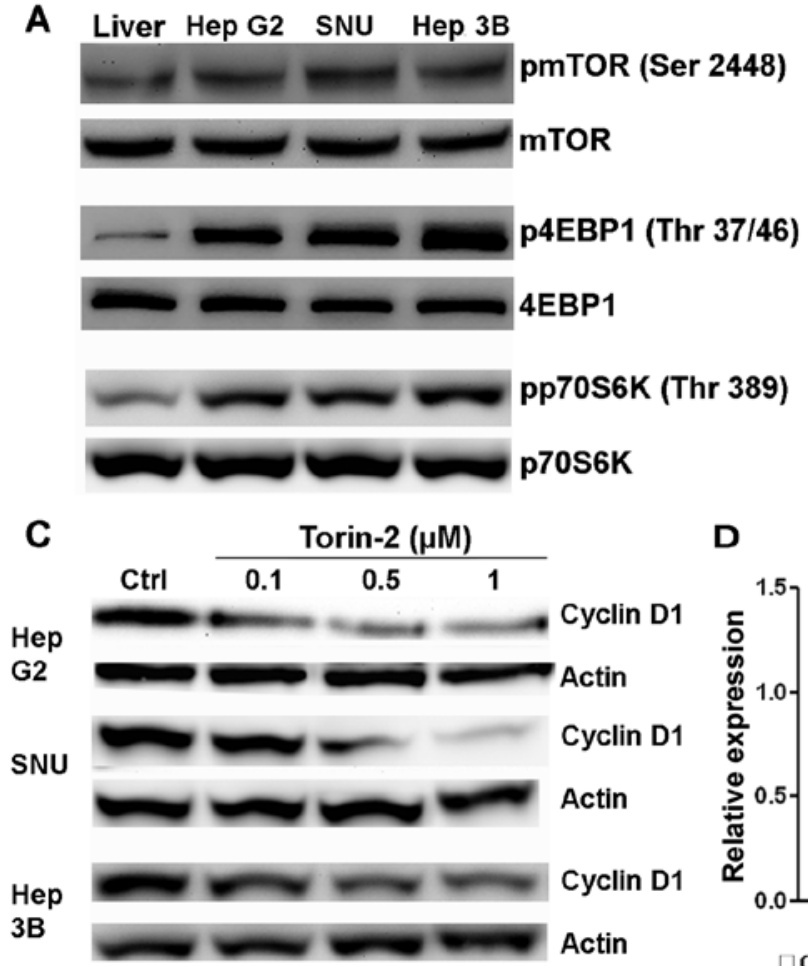

D
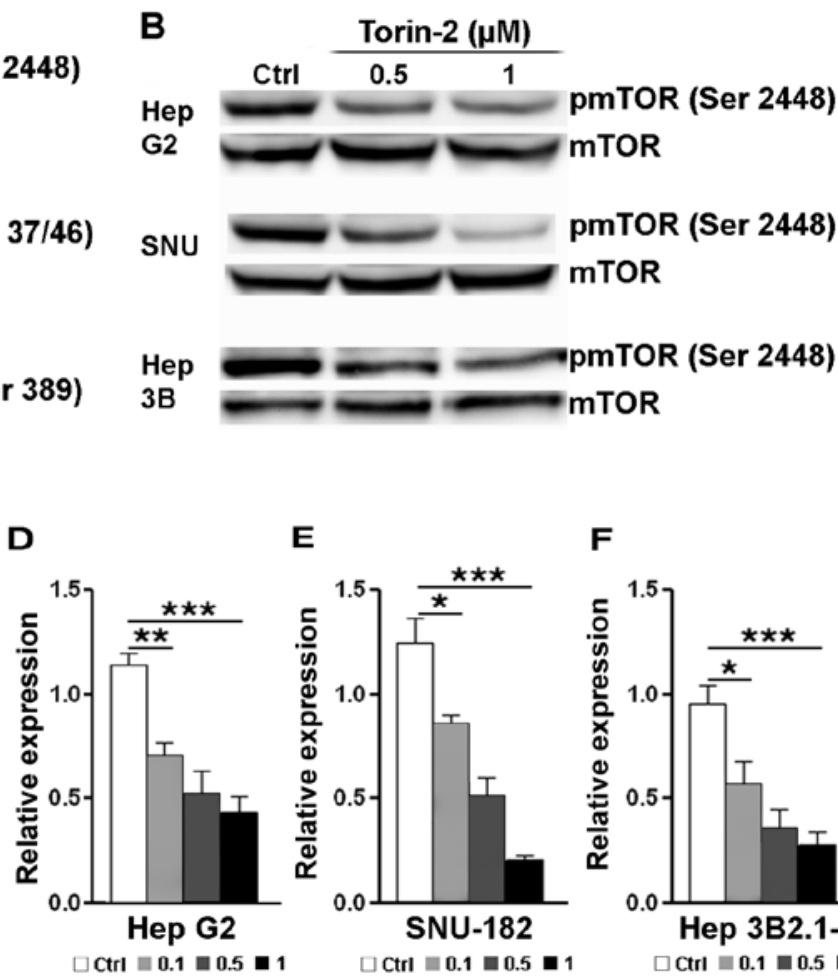

E

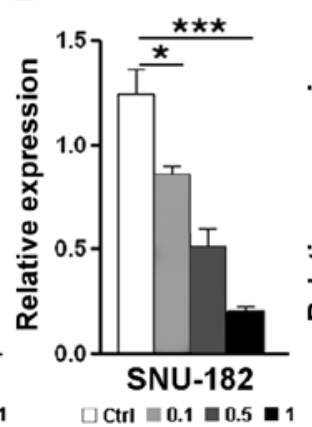

$\mathbf{F}$

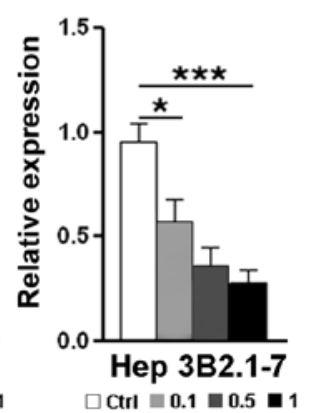

Figure 2. Torin-2 inhibits mTOR activation and decreases cyclin D1 expression. (A) Activating phosphorylation of mTOR, 4EBP1 and p70S6K in normal liver tissue and HCC cell lines. A representative image of two independent experiments is shown. (B) Torin- 2 decreased activating phosphorylation of mTOR in the HCC cell lines on day 3 after treatment. A representative image of three independent experiments is shown. (C) Torin- 2 dose-dependently downregulated cyclin D1 expression in the HCC cell lines on day 3 after treatment. A representative image of four independent experiments is shown. (D-F) Quantification of cyclin D1 expression in the HCC cell lines. $\mathrm{n}=4$ /group. SNU, SNU-182; Hep 3B, Hep 3B2.1-7; Ctrl, vehicle control; ${ }^{*} \mathrm{P}<0.05$, ${ }^{* *} \mathrm{P}<0.01$ and ${ }^{* * *} \mathrm{P}<0.001$. mTOR, mammalian target of rapamycin; $\mathrm{HCC}$, hepatocellular carcinoma.

sition in cell cycle progression (22). Thus, this reduction was consistent with our previous data showing $\mathrm{G}_{0} / \mathrm{G}_{1}$ arrest and a decreased $\mathrm{S}$ phase population of cancer cells. Furthermore, cyclin D translation is regulated by the mTORC1 signaling pathway (23). Hence, a reduced cyclin D1 level was possibly due to the mTORC1 blockage by Torin-2. Collectively, our data indicated that Torin-2 indeed inhibits mTORC1 signaling in HCC cells.

Torin-2 induces cancer cell apoptosis. An initial reduction in cell number within 3 days after high-dose Torin-2 treatment (Fig. 1A-C) indicated that Torin-2 is cytotoxic to cancer cells. To test this hypothesis, we determined the extent of cell apoptosis with Annexin V and PI staining. As shown in Fig. 3, the percentage of Annexin-V-positive cells was markedly increased with increasing Torin- 2 concentrations, suggesting that Torin-2 induced cancer cell apoptosis in a dose-dependent manner. Surprisingly, necrosis was not profound even in the treated HCC cells. Less than $6 \%$ of cells underwent necrosis in the Torin-2-treated groups, although the moderate increase in necrosis was statistically significant. The weak necrosis induction by Torin- 2 may be beneficial for future cancer therapy, since necrosis would trigger a severe inflammatory response, while apoptosis would not.

Torin-2 upregulates autophagy in liver cancer cells. A previous study showed that Torin-2 promotes autophagy in leukemic cell lines (24). Since autophagy is closely related to cancer cell survival, we ascertained whether Torin-2 induced autophagy in liver cancer cells by detecting autophagic marker expression. As shown in Fig. 4, in all three cell lines, Torin-2 significantly elevated LC3B conversion from LC3B-I to LC3B-II, demonstrated by higher LC3B-II levels. Beclin-1, which initiates autophagy, was also upregulated by Torin-2. p62, which is degraded by autophagosomes, reflects the extent of autophagic protein degradation. The p62 level was markedly reduced following Torin-2 treatment. Collectively, our data indicated that Torin-2 effectively promoted autophagy in HCC cells.

Torin-2-induced autophagy inhibits the proliferation and survival of liver cancer cells. To confirm that Torin-2-induced autophagy is the cause of the inhibition of proliferation and increased apoptosis of liver cancer cells, we blocked autophagy with the well-known autophagy inhibitor chloroquine, which is a lysosomotropic agent preventing endosomal acidification (25). Chloroquine significantly upregulated the LC3B-II level due to inhibition of autophagic protein degradation. The p62 level was also enhanced for the same reason (Fig. 5A), suggesting that autophagy was indeed inhibited. Cell proliferation assay with Ki67 staining showed that chloroquine partially restored cell proliferation in the presence of Torin-2 in all three cell lines (Fig. 5B), suggesting that Torin-2-induced autophagy was at least one of the causes of cell proliferation inhibition. Apoptosis assay with Annexin-V staining indicated that chloroquine significantly reduced the percentage of 
A
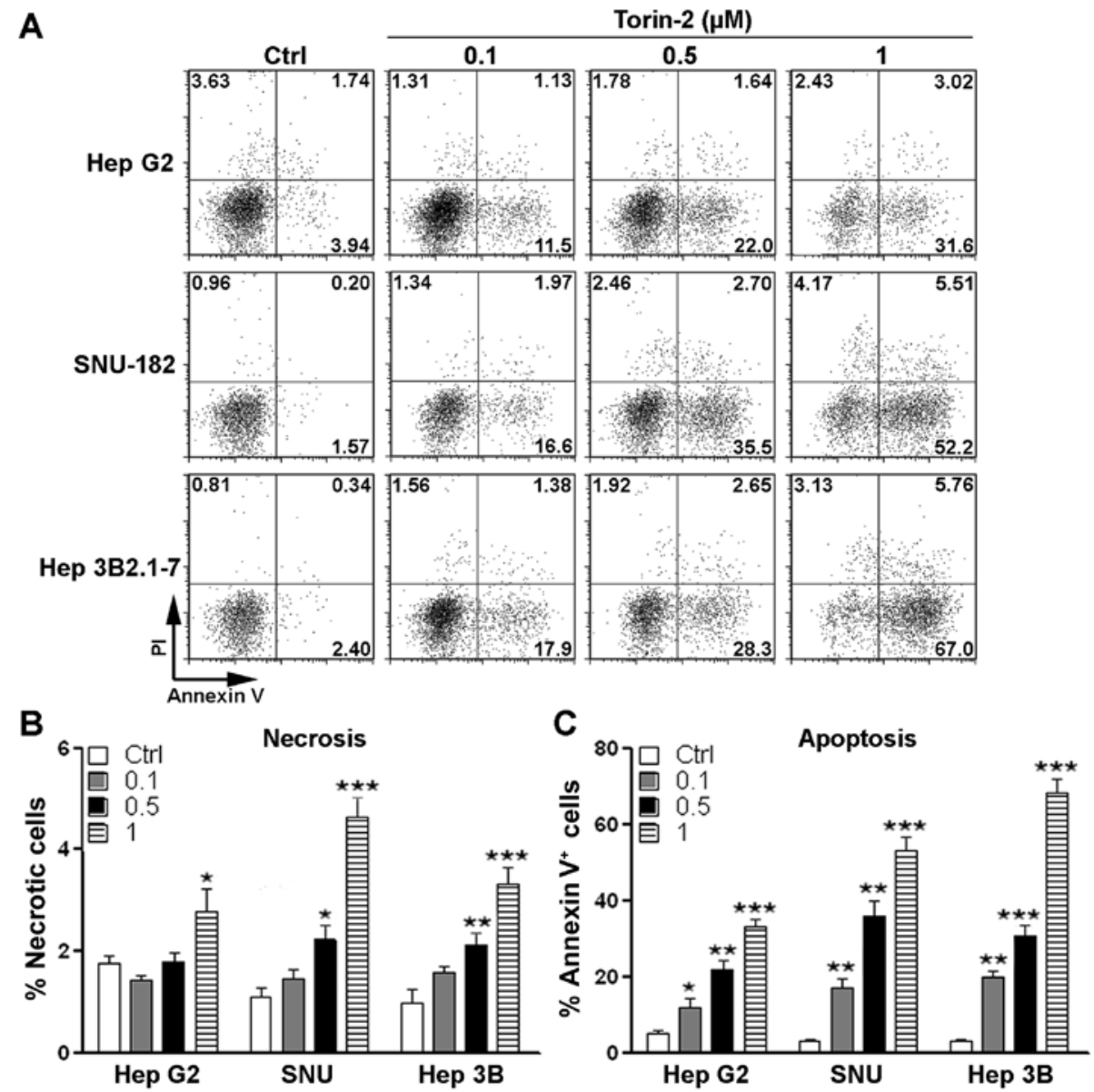

Figure 3. Torin-2 induces apoptosis of the HCC cell lines. (A) Representative dot plots of Annexin V and PI staining in the HCC cell lines on day 3 after treatment. Numbers in the quadrants are the percentages of each cell population. (B and C) Quantification of necrosis and apoptosis. $\mathrm{n}=6 / \mathrm{group}$. SNU, SNU-182; Hep 3B, Hep 3B2.1-7; Ctrl, vehicle control; ${ }^{*} \mathrm{P}<0.05,{ }^{* *} \mathrm{P}<0.01$ and ${ }^{* * * *} \mathrm{P}<0.001$, in comparison with the vehicle control; HCC, hepatocellular carcinoma.
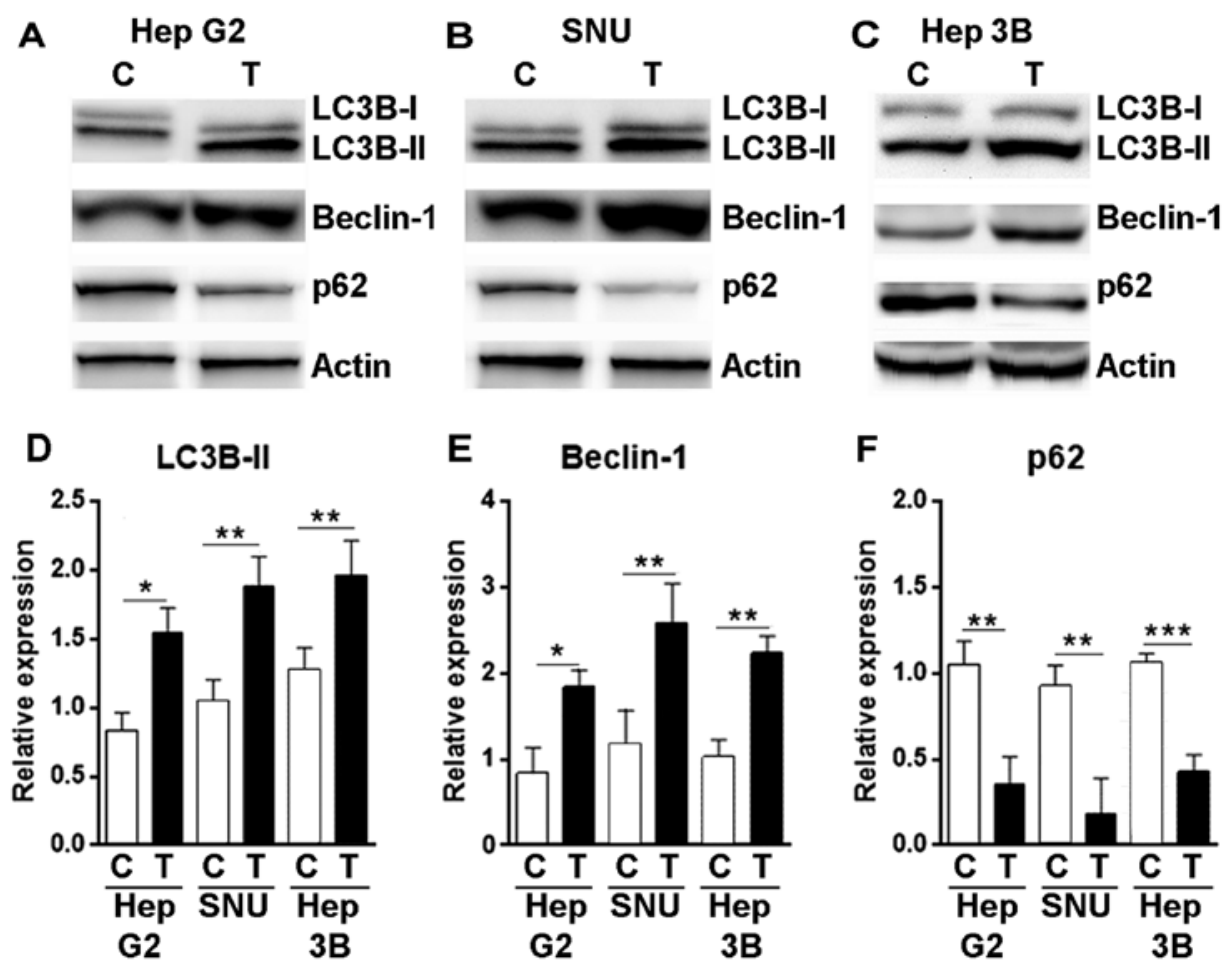

Figure 4. Torin-2 induces autophagy in the HCC cell lines. (A-C) Expression of LC3B, Beclin-1 and p62 in Hep G2 (A), SNU-182 (B) and Hep 3B2.1-7 (C) cells

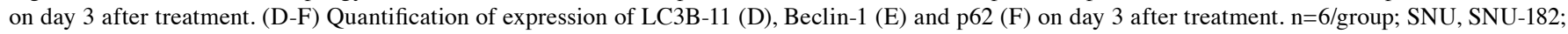
Hep 3B, Hep 3B2.1-7; C, vehicle control; T, $1 \mu \mathrm{M}$ Torin-2-treated cells; ${ }^{*} \mathrm{P}<0.05,{ }^{* * *} \mathrm{P}<0.01$ and ${ }^{* * *} \mathrm{P}<0.001$. HCC, hepatocellular carcinoma. 


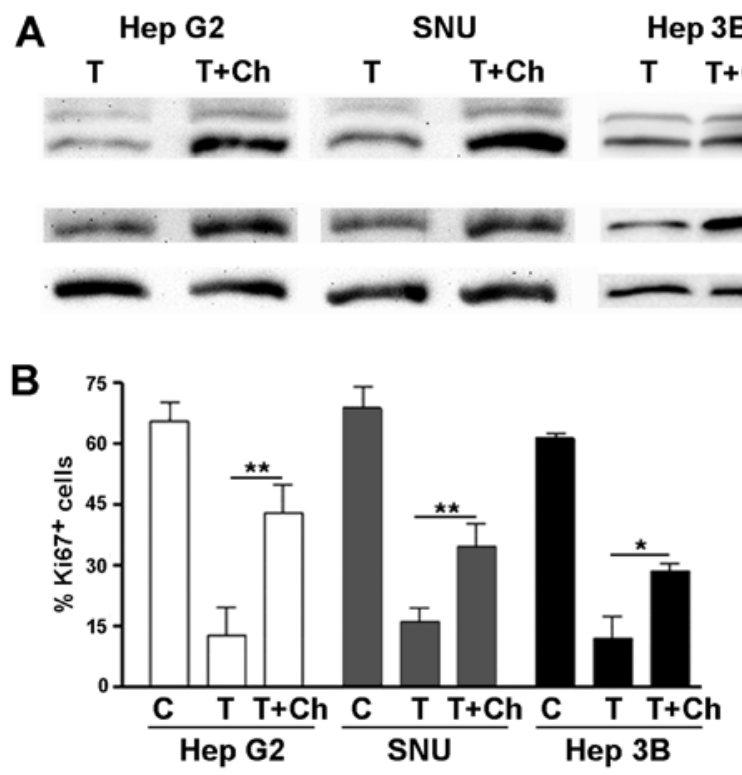

\section{LC3B-I \\ LC3B-II \\ p62 \\ Actin}

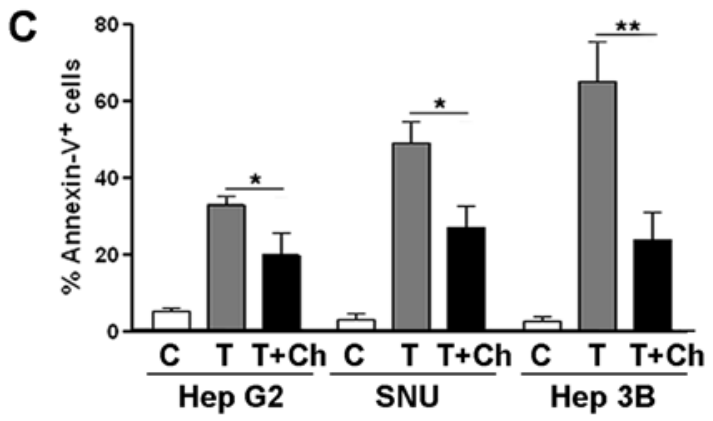

Figure 5. Torin-2-induced autophagy contributes to proliferation inhibition and apoptosis of HCC cells. (A) Expression of LC3B and p62 in HCC cells after treatment with Torin-2 in the presence or absence of chloroquine (Ch). A representative image of three independent experiments is shown. (B) Quantification of Ki67 staining of HCC cells. (C) Quantification of apoptosis of the HCC cells. n=5/group. SNU, SNU-182; Hep 3B, Hep 3B2.1-7; C, vehicle control; T, $1 \mu$ M Torin-2; $\mathrm{T}+\mathrm{Ch}, 1 \mu \mathrm{M}$ Torin-2 with $10 \mu \mathrm{M}$ chloroquine; ${ }^{*} \mathrm{P}<0.05$ and ${ }^{* *} \mathrm{P}<0.01$. HCC, hepatocellular carcinoma.
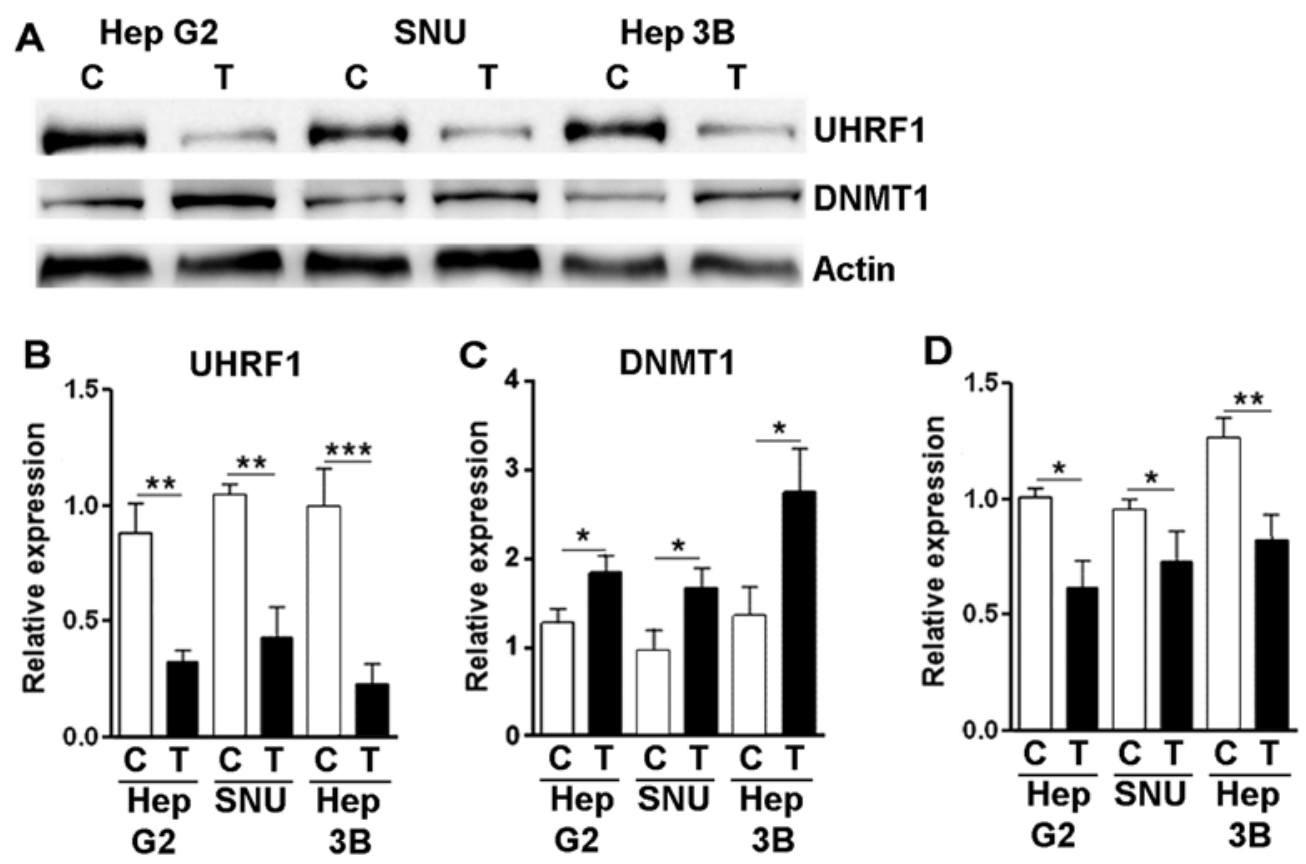

Figure 6. Torin-2 downregulates UHRF1 expression and upregulates DNMT1 expression. (A) Expression of UHRF1 and DNMT1 in the HCC cells after treatment with Torin-2. A representative image of four independent experiments is shown. (B and C) Quantification of expression of UHRF1 (B) and DNMT1 (C) in the HCC cells after treatment with Torin-2. (D) UHRF1 mRNA level as detected by real-time PCR. n=4/group. SNU, SNU-182; Hep 3B, Hep 3B2.1-7; $\mathrm{C}$, vehicle control; $\mathrm{T}, 1 \mu \mathrm{M}$ Torin $-2 ;{ }^{*} \mathrm{P}<0.05,{ }^{* *} \mathrm{P}<0.01$ and ${ }^{* * *} \mathrm{P}<0.001$. HCC, hepatocellular carcinoma.

apoptotic cells in the presence of Torin-2 (Fig. 5C), suggesting that Torin-2-induced autophagy indeed triggers apoptosis.

Torin-2 downregulates UHRF1 expression in liver cancer cells. UHRF1 has been identified as an important regulator for DNA methylation during DNA replication. Recently it was reported that UHRF1 is responsible for hepatocellular carcinogenesis (20). The relationship between mTORC1 signaling and UHRF1 expression has not yet been elucidated. We detected the UHRF1 protein level using western blot analysis and found that Torin-2 treatment significantly decreased UHRF1 expression in the HCC cell lines (Fig. 6A and B). Consistently, the expression of DNMT1, which is destabilized by URHF1, was upregulated in the presence of Torin-2 (Fig. 6A and C). We further determined the mRNA level of URHF1 using real-time PCR. Our data showed that Torin-2 treatment moderately decreased URHF1 mRNA in these cells (Fig. 6D), suggesting that Torin-2 inhibited URHF1 transcription, or destabilized 
URHF1 mRNA. However, it is still not clear whether Torin-2 decreased URHF1 mRNA through mTORC1 signaling, since few studies have reported that mTORC1 signaling can regulate gene transcription. Further studies are needed to dissect the mechanisms responsible for the reduction in URHF1 mRNA.

\section{Discussion}

HCC is the third leading cause of cancer-related mortality worldwide. The incidence of this disease is increasing and it is one of the key indications for liver transplantation. It is the most common primary liver tumor, which is notoriously resistant to systemic therapies and often recurs even after aggressive local therapies. HCC initiation and progression are complicated, involving alterations in multiple signal transduction pathways and subsequent cellular behavior change.

In the present study, we tested the effect of Torin-2 on the proliferation and survival of three HCC cell lines. Torin-2 is a novel ATP-competitive inhibitor of mTOR. mTOR is a highly conserved serine/threonine protein kinase that serves as a central regulator of cell growth, survival and autophagy. Deregulation of the PI3K/Akt/mTOR signaling pathway commonly occurs in cancer (26). Given its importance in cell growth and metabolism, mTOR also plays a pivotal role in HCC (6). mTORC1 and mTORC2 pathways, including pRPS6, p-AKT, IGF-1R and RICTOR are upregulated in $40-50 \%$ of HCCs (27). Moreover, an upregulation of mTOR signaling is frequently observed in cholangiocarcinoma, the second most common primary cancer of the liver (5). Activation of the mTOR pathway in HCC is associated with less differentiated tumors, poor prognosis and earlier recurrence independently of the underlying etiology of liver cancer. We tested the mTORC1 activation status in these HCC cell lines and found that all had upregulated mTORC1 signaling, in comparison with the normal mouse liver tissue. Thus, it is highly possible that mTORC1 activation is critical for the initiation and/or maintenance of carcinogenic features in these HCC cells. Therefore, blocking this pathway is an attractive strategy for HCC treatment. Until recently, several inhibitors targeting mTOR have been studied for their efficacy in tumor therapy, including rapamycin (28) and its derivatives everolimus (11), temsirolimus (29-31) and deforolimus (32). In contrast to these first generation mTOR inhibitors, Torin-2 inhibits mTOR kinase and mTORC1 signaling activities in a sustained manner suggestive of a slow dissociation from the kinase (14). It has been reported that cancer cell treatment with Torin- 2 for $24 \mathrm{~h}$ resulted in a prolonged block in negative feedback and consequent T308 phosphorylation on Akt (14). These effects were associated with strong growth inhibition in vitro. To our knowledge, however, Torin-2 has not been assessed for treating $\mathrm{HCC}$ in vitro or in vivo.

Our in vitro study indicated that Torin-2 profoundly reduced $\mathrm{HCC}$ cell growth in a dose-dependent manner. This growth inhibition was attributed to $G_{0} / G_{1}$ phase arrest in the cell cycle. Consistently, cyclin D expression was downregulated by Torin-2. Cyclin D is the partner of cyclin-dependent kinases CDK4 and CDK6. CDK4 and CDK6 drive the transition of $\mathrm{G}_{1}$ to $S$ phase in the cell cycle. Activity of the cyclin-dependent CDK4 and CDK6 is regulated by T-loop phosphorylation, by the abundance of cyclin D $(33,34)$. Thus, the downregulation of cyclin D could be the reason for $\mathrm{G}_{0} / \mathrm{G}_{1}$ arrest, preventing cells from entering the DNA synthesis phase. Moreover, the translation of cyclin D protein is tightly regulated by mTORC1 signaling, since mTORC1 activates 4EBP1 and p70S6K, which both are important participants in the protein synthesis machinery (35). We indeed confirmed that Torin-2 inhibited the activating phosphorylation of mTOR. Therefore, it is highly possible that the blockage of mTORC1 activation by Torin- 2 decreased the translation of cyclin $\mathrm{D}$, consequently reducing the activity of CDK4 and CDK6 to prevent cell entry to the $S$ phase. However, we did not test whether other cell cycle regulators, such as CDK inhibitors and cdc2 are influenced by Torin-2. In addition, it is still not clear whether Torin- 2 impacts autocrine/paracrine factors which profoundly modulate HCC initiation and progression, such as IGF-1, IGF-2, IGFBP-3, TGF- $\alpha$, TGF- $\beta$, EGF and FGF. Further studies are needed to include or exclude the effects of Torin- 2 on these regulatory factors.

In addition, our research revealed that Torin- 2 can induce HCC cell apoptosis, which could also contribute to the inhibition of HCC cell growth. Furthermore, Torin-2-induced apoptosis is closely related to autophagy, which is a crucial mechanism underlying tumor cell metabolism and survival. Autophagy is a homeostatic, catabolic degradation process whereby cellular proteins and organelles are engulfed by autophagosomes, digested in lysosomes and recycled to sustain cellular metabolism (36). Autophagy genes are frequently mono-allelically deleted, silenced or mutated in human tumors, resulting in an environment of increased oxidative stress that is conducive to DNA damage, genomic instability and tumor progression. It has been known that autophagy can lead to autophagic cell death in HCCs (37-40). However, previous research has also revealed that autophagy induction could also be beneficial for tumor cell growth (41-44). Thus, the extent of autophagy may be very important to decide the fate of HCC cells. To our knowledge, how much autophagy induction is needed for tumor growth or death is still poorly understood. Previous studies on rapamycin found that rapamycin-induced autophagy could be either pro-apoptotic (45) or anti-apoptotic (46). The Torin-2 concentration we used could have been high enough to cause autophagic cell death of HCC cells. It will be interesting to test whether lower doses of Torin-2 induce weaker autophagy and promote HCC cell survival. If this was the case, Torin-2 dosage must be taken into account in future therapeutic applications for tumors.

What was unexpected in our research was the downregulation of UHRF1 by Torin-2. UHRF1 plays an essential role in DNA methylation by recognizing hemimethylated DNA generated during DNA replication and then by recruiting DNMT1 to ensure faithful maintenance of DNA-methylation patterns in daughter cells $(47,48)$. DNA hypomethylation contributes to oncogenesis through multiple mechanisms, including chromosomal instability, derepression of imprinted genes, retrotransposon activation and aberrant gene expression, including induction of oncogenes (20). The precise mechanism by which DNA hypomethylation causes cancer remains elusive. Both apoptosis and senescence serve to limit the propagation of cells with aberrant DNA methylation. DNMT1 and UHRF1 are significantly altered across cancer types. Change in the expression of UHRF1 is sufficient to alter the cancer 
cell methylome and drive carcinogenesis. However, there is no evidence suggesting the relationship between mTORC1 signaling and DNA methylation. To our knowledge we are the first to report that mTORC1 signaling can regulate the expression of UHRF1. Our data indicated that the downregulation of UHRF1 by Torin- 2 was at least partially due to the reduced UHRF1 mRNA levels. Since mTORC1 classically modulates metabolism and protein synthesis, it is plausible to speculate that mTORC1 regulates the translation of unknown factors which subsequently influences the transcription of UHRF1. However, we cannot exclude the possibility that Torin-2 suppresses UHRF1 expression independently of mTORC1. The mechanisms modulating UHRF1 expression are still very poorly elucidated. Further studies will be performed to dissect the signaling pathways causing this change. We confirmed that with decreased UHRF1 expression, expression of DNMT1 was upregulated. This may be due to the fact that destabilization of DNMT1 by UHRF1 is compromised as UHRF1 itself is downregulated.

Collectively, the present study demonstrated the effect of Torin-2 on the inhibition of HCC cell proliferation and survival. The inhibitory efficacy can be ascribed to blockage of mTORC1 signaling, subsequently enhancing autophagy and leading to autophagic cell death. Meanwhile, blockage of mTORC1 also suppressed the cell entry into the $\mathrm{S}$ phase, thus causing $\mathrm{G}_{0} / \mathrm{G}_{1}$ arrest. Torin-2 also downregulated the expression of UHRF1, which consequently impacted the oncogenesis of HCC. Our research provides new clues in the search for therapeutic strategies for HCC.

\section{References}

1. Olsen SK, Brown RS and Siegel AB: Hepatocellular carcinoma: Review of current treatment with a focus on targeted molecular therapies. Therap Adv Gastroenterol 3: 55-66, 2010.

2. Breuhahn K, Longerich T and Schirmacher P: Dysregulation of growth factor signaling in human hepatocellular carcinoma. Oncogene 25: 3787-3800, 2006.

3. Moeini A, Cornellà $H$ and Villanueva A: Emerging signaling pathways in hepatocellular carcinoma. Liver Cancer 1: 83-93, 2012.

4. Sanz-Cameno P, Trapero-Marugán M, Chaparro M, Jones EA and Moreno-Otero R: Angiogenesis: From chronic liver inflammation to hepatocellular carcinoma. J Oncol 2010: 272170, 2010.

5. Matter MS, Decaens T, Andersen JB and Thorgeirsson SS: Targeting the mTOR pathway in hepatocellular carcinoma: Current state and future trends. J Hepatol 60: 855-865, 2014.

6. Zhou Q, Lui VW and Yeo W: Targeting the PI3K/Akt/mTOR pathway in hepatocellular carcinoma. Future Oncol 7: 1149-1167, 2011.

7. Newell P, Toffanin S, Villanueva A, Chiang DY, Minguez B, Cabellos L, Savic R, Hoshida Y, Lim KH, Melgar-Lesmes P, et al: Ras pathway activation in hepatocellular carcinoma and anti-tumoral effect of combined sorafenib and rapamycin in vivo. J Hepatol 51: 725-733, 2009.

8. Guertin DA and Sabatini DM: Defining the role of mTOR in cancer. Cancer Cell 12: 9-22, 2007.

9. Wörns MA and Galle PR: HCC therapies - lessons learned. Nat Rev Gastroenterol Hepatol 11: 447-452, 2014.

10. Martelli AM, Chiarini F, Evangelisti C, Cappellini A, Buontempo F, Bressanin D, Fini M and McCubrey JA: Two hits are better than one: Targeting both phosphatidylinositol 3-kinase and mammalian target of rapamycin as a therapeutic strategy for acute leukemia treatment. Oncotarget 3: 371-394, 2012.

11. Zhu AX, Kudo M, Assenat E, Cattan S, Kang YK, Lim HY, Poon RT, Blanc JF, Vogel A, Chen CL, et al: Effect of everolimus on survival in advanced hepatocellular carcinoma after failure of sorafenib: The EVOLVE-1 randomized clinical trial. JAMA 312: 57-67, 2014.
12. Zhou Q, Wong CH, Lau CP, Hui CW, Lui VW, Chan SL and Yeo W: Enhanced antitumor activity with combining effect of mTOR inhibition and microtubule stabilization in hepatocellular carcinoma. Int J Hepatol 2013: 103830, 2013.

13. Liu Q, Wang J, Kang SA, Thoreen CC, Hur W, Ahmed T, Sabatini DM and Gray NS: Discovery of 9-(6-aminopyridin-3yl)-1-(3-(trifluoromethyl)phenyl)benzo[h][1,6]naphthyridin-2(1H) - one (Torin2) as a potent, selective, and orally available mammalian target of rapamycin (mTOR) inhibitor for treatment of cancer. J Med Chem 54: 1473-1480, 2011.

14. Liu Q, Xu C, Kirubakaran S, Zhang X, Hur W, Liu Y, Kwiatkowski NP, Wang J, Westover KD, Gao P, et al: Characterization of Torin2, an ATP-competitive inhibitor of mTOR, ATM, and ATR. Cancer Res 73: 2574-2586, 2013.

15. Ahmed M, Hussain AR, Bavi P, Ahmed SO, Al Sobhi SS Al-Dayel F, Uddin S and Al-Kuraya KS: High prevalence of mTOR complex activity can be targeted using Torin 2 in papillary thyroid carcinoma. Carcinogenesis 35: 1564-1572, 2014.

16. Bonder MJ, Kasela S, Kals M, Tamm R, Lokk K, Barragan I, Buurman WA, Deelen P, Greve JW, Ivanov M, et al: Genetic and epigenetic regulation of gene expression in fetal and adult human livers. BMC Genomics 15: 860, 2014.

17. Robertson KD: DNA methylation, methyltransferases, and cancer. Oncogene 20: 3139-3155, 2001.

18. Bianchi $\mathrm{C}$ and Zangi R: UHRF1 discriminates against binding to fully-methylated $\mathrm{CpG}$-Sites by steric repulsion. Biophys Chem 171: 38-45, 2013.

19. Bashtrykov P, Jankevicius G, Jurkowska RZ, Ragozin S and Jeltsch A: The UHRF1 protein stimulates the activity and specificity of the maintenance DNA methyltransferase DNMT1 by an allosteric mechanism. J Biol Chem 289: 4106-4115, 2014.

20. Mudbhary R, Hoshida Y, Chernyavskaya Y, Jacob V, Villanueva A, Fiel MI, Chen X, Kojima K, Thung S, Bronson RT, et al: UHRF1 overexpression drives DNA hypomethylation and hepatocellular carcinoma. Cancer Cell 25: 196-209, 2014.

21. Pi JT, Lin Y, Quan Q, Chen LL, Jiang LZ, Chi W and Chen HY: Overexpression of UHRF1 is significantly associated with poor prognosis in laryngeal squamous cell carcinoma. Med Oncol 30: 613, 2013.

22. Choi YJ, Li X, Hydbring P, Sanda T, Stefano J, Christie AL, Signoretti S, Look AT, Kung AL, von Boehmer H, et al: The requirement for cyclin $\mathrm{D}$ function in tumor maintenance. Cancer Cell 22: 438-451, 2012.

23. Averous J, Fonseca BD and Proud CG: Regulation of cyclin D1 expression by mTORC1 signaling requires eukaryotic initiation factor 4E-binding protein 1. Oncogene 27: 1106-1113, 2008.

24. Simioni C, Cani A, Martelli AM, Zauli G, Tabellini G, McCubrey J, Capitani S and Neri LM: Activity of the novel mTOR inhibitor Torin-2 in B-precursor acute lymphoblastic leukemia and its therapeutic potential to prevent Akt reactivation. Oncotarget 5: 10034-10047, 2014.

25. Kimura T, Takabatake Y, Takahashi A and Isaka Y: Chloroquine in cancer therapy: A double-edged sword of autophagy. Cancer Res 73: 3-7, 2013.

26. Polivka J Jr and Janku F: Molecular targets for cancer therapy in the PI3K/AKT/mTOR pathway. Pharmacol Ther 142: 164-175, 2014.

27. Villanueva A, Chiang DY, Newell P, Peix J, Thung S, Alsinet C, Tovar V, Roayaie S, Minguez B, Sole M, et al: Pivotal role of mTOR signaling in hepatocellular carcinoma. Gastroenterology 135: 1972-1983, 2008.

28. Ashworth RE and Wu J: Mammalian target of rapamycin inhibition in hepatocellular carcinoma. World J Hepatol 6: 776-782, 2014.

29. Knox JJ, Qin R, Strosberg JR, Tan B,Kaubisch A, El-Khoueiry AB, Bekaii-Saab TS, Rousey SR, Chen HX, Erlichman C: A phase II trial of bevacizumab plus temsirolimus in patients with advanced hepatocellular carcinoma. Invest New Drugs 33: 241-246, 2014.

30. Kelley RK, Nimeiri HS, Munster PN, Vergo MT, Huang Y, Li CM, Hwang J, Mulcahy MF, Yeh BM, Kuhn P, et al: Temsirolimus combined with sorafenib in hepatocellular carcinoma: A phase I dose-finding trial with pharmacokinetic and biomarker correlates. Ann Oncol 24: 1900-1907, 2013.

31. Zhou Q, Lui VW, Lau CP, Cheng SH, Ng MH, Cai Y, Chan SL and Yeo W: Sustained antitumor activity by co-targeting mTOR and the microtubule with temsirolimus/vinblastine combination in hepatocellular carcinoma. Biochem Pharmacol 83: 1146-1158, 2012.

32. Wang Z, Jin W, Jin $\mathrm{H}$ and Wang $\mathrm{X}$ : $\mathrm{mTOR}$ in viral hepatitis and hepatocellular carcinoma: Function and treatment. Biomed Res Int 2014: 735672, 2014. 
33. Bockstaele L, Kooken H, Libert F, Paternot S, Dumont JE, de Launoit Y, Roger PP and Coulonval K: Regulated activating Thr172 phosphorylation of cyclin-dependent kinase 4(CDK4): Its relationship with cyclins and CDK 'inhibitors'. Mol Cell Biol 26: 5070-5085, 2006.

34. Choi YJ and Anders L: Signaling through cyclin D-dependent kinases. Oncogene 33: 1890-1903, 2014.

35. Takuwa N,Fukui Y and Takuwa Y: Cyclin D1 expression mediated by phosphatidylinositol 3-kinase through mTOR-p70(S6K)independent signaling in growth factor-stimulated NIH 3T3 fibroblasts. Mol Cell Biol 19: 1346-1358, 1999.

36. Yang ZJ, Chee CE, Huang S and Sinicrope FA: The role of autophagy in cancer: Therapeutic implications. Mol Cancer Ther 10: 1533-1541, 2011.

37. Sui X, Chen R, Wang Z, Huang Z, Kong N, Zhang M, Han W, Lou F, Yang J, Zhang Q, et al: Autophagy and chemotherapy resistance: A promising therapeutic target for cancer treatment. Cell Death Dis 4: e838,2013.

38. Li P, Du Q, Cao Z, Guo Z, Evankovich J, Yan W, Chang Y, Shao L, Stolz DB, Tsung A, et al: Interferon- $\gamma$ induces autophagy with growth inhibition and cell death in human hepatocellular carcinoma (HCC) cells through interferon-regulatory factor-1 (IRF-1). Cancer Lett 314: 213-222, 2012.

39. Tai WT, Shiau CW, Chen HL, Liu CY, Lin CS, Cheng AL, Chen PJ and Chen KF: Mcl-1-dependent activation of Beclin 1 mediates autophagic cell death induced by sorafenib and SC-59 in hepatocellular carcinoma cells. Cell Death Dis 4: e485, 2013.

40. Allen KD, Mata BA, Gabr MA, Huebner JL, Adams SB Jr, Kraus VB, Schmitt DO and Setton LA: Kinematic and dynamic gait compensations resulting from knee instability in a rat model of osteoarthritis. Arthritis Res Ther 14: R78, 2012.
41. Zhou Y, Sun K, Ma Y, Yang H, Zhang Y, Kong X and Wei L: Autophagy inhibits chemotherapy-induced apoptosis through downregulating Bad and Bim in hepatocellular carcinoma cells. Sci Rep 4: 5382, 2014

42. Guo XL, Li D, Sun K, Wang J, Liu Y, Song JR, Zhao QD, Zhang SS, Deng WJ, Zhao X, et al: Inhibition of autophagy enhances anticancer effects of bevacizumab in hepatocarcinoma. J Mol Med Berl 91: 473-483, 2013.

43. Yang PM, Liu YL, Lin YC, Shun CT, Wu MS and Chen CC: Inhibition of autophagy enhances anticancer effects of atorvastatin in digestive malignancies. Cancer Res 70: 7699-7709, 2010.

44. Li J, Yang B, Zhou Q, Wu Y, Shang D, Guo Y, Song Z, Zheng Q and Xiong J: Autophagy promotes hepatocellular carcinoma cell invasion through activation of epithelial-mesenchymal transition. Carcinogenesis 34: 1343-1351, 2013.

45. Li X, Wu D, Shen J, Zhou M and Lu Y: Rapamycin induces autophagy in the melanoma cell line M14 via regulation of the expression levels of Bcl-2 and Bax. Oncol Lett 5: 167-172, 2013.

46. Ravikumar B,BergerZ, Vacher C, O'Kane CJ and Rubinsztein DC Rapamycin pre-treatment protects against apoptosis. Hum Mol Genet 15: 1209-1216, 2006.

47. Unoki M: Current and potential anticancer drugs targeting members of the UHRF1 complex including epigenetic modifiers. Recent Patents Anticancer Drug Discov 6: 116-130, 2011.

48. Bronner C, Fuhrmann G, Chédin FL, Macaluso $M$ and Dhe-Paganon S: UHRF1 links the histone code and DNA methylation to ensure faithful epigenetic memory inheritance. Genet Epigenet 2009: 29-36, 2010. 